\title{
Deep sternal wound infections: Evidence for prevention, treatment, and reconstructive surgery
}

\author{
Luigi Schiraldi ${ }^{1,2}$, Gaby Jabbour ${ }^{2,3}$, Paolo Centofanti ${ }^{4}$, Salvatore Giordano ${ }^{5}$, Etienne Abdelnour ${ }^{6}$, \\ Michel Gonzalez ${ }^{6}$, Wassim Raffoul ${ }^{2}$, Pietro Giovanni di Summa ${ }^{2}$ \\ ${ }^{1}$ Department of Surgery, Hospital du Valais, Sion; ${ }^{2}$ Department of Plastic and Reconstructive Surgery, Lausanne University Hospital \\ (CHUV), Lausanne, Switzerland; ${ }^{3}$ Department of Surgery, Hamad General Hospital, Doha, Qatar; ${ }^{4}$ Department of Cardiac Surgery, \\ Mauriziano Hospital, Turin, Italy; ${ }^{5}$ Department of Plastic and General Surgery, Turku University Hospital, Turku, Finland; ${ }^{6}$ Department of \\ Thoracic Surgery, Lausanne University Hospital (CHUV), Lausanne, Switzerland
}

\begin{abstract}
Median sternotomy is the most popular approach in cardiac surgery. Post-sternotomy wound complications are rare, but the occurrence of a deep sternal wound infection (DSWI) is a catastrophic event associated with higher morbidity and mortality, longer hospital stays, and increased costs. A literature review was performed by searching PubMed from January 1996 to August 2017 according to the guidelines in the Preferred Reporting Items for Systematic Reviews and Meta-Analyses statement. The following keywords were used in various combinations: DSWI, post-sternotomy complication, and sternal reconstruction. Thirty-nine papers were included in our qualitative analysis, in which each aspect of the DSWI-related care process was analyzed and compared to the actual standard of care. Plastic surgeons are often involved too late in such clinical scenarios, when previous empirical treatments have failed and a definitive reconstruction is needed. The aim of this comprehensive review was to create an up-to-date operative flowchart to prevent and properly treat sternal wound infection complications after median sternotomy.
\end{abstract}

Keywords Sternum / Infection / Reconstructive surgical procedure / Sternotomy

\author{
Correspondence: \\ Pietro Giovanni di Summa \\ Department of Plastic and \\ Reconstructive Surgery, Lausanne \\ University Hospital (CHUV), Rue du \\ Bugnon 46, Lausanne 1005, \\ Switzerland \\ Tel: +41-21-314-25-25 \\ Fax: +41-21-314-25-30 \\ E-mail: pietro.di-summa@chuv.ch
}

The authors are thankful to Mr. Marco Schmid, Arch. and Mrs. Carol De Simio for their valuable advice and help with illustrations.

Received: 3 Sep $2018 \bullet$ Revised: 14 Jun $2019 \bullet$ Accepted: 3 Jul 2019

pISSN: 2234-6163 • elSSN: 2234-6171 • https://doi.org/10.5999/aps.2018.01151 • Arch Plast Surg 2019;46:291-302

\section{INTRODUCTION}

Despite the increasingly common use of minimally invasive techniques, the vast majority of cardiac operations still use median sternotomy, as it is easily and expeditiously performed, and provides excellent exposure $[1,2]$. When compared to other surgical approaches such as laparotomy, post-sternotomy complications are infrequent. However, deep sternal wound infection (DSWI) can be a catastrophic complication, as it is associated with prolonged hospital stays, long-term antibiotic therapy, multiple surgical procedures, higher mortality and morbidity, and increased patient suffering and costs $[3,4]$. The reported incidence of DSWI ranges between $0.8 \%$ and $6.0 \%[5,6]$. The current literature consists mainly of small case series with a low level of evidence, without systematic reviews and meta-analyses. Considering the lack of established treatment protocols, the aim of this review was to provide an up-to-date evidence synthesis. The resulting operative flowchart is meant to be useful to both cardio-thoracic and plastic surgery units by providing guidance on how to treat post-sternotomy complications properly. A full 
understanding of the etiology, prevention, and surgical reconstruction of DSWI is crucial for proper treatment.

\section{METHODS}

A systematic search of the literature was performed by searching PubMed from January 1996 to August 2017 according to the Preferred Reporting Items for Systematic Reviews and MetaAnalyses statement (Fig. 1) [7]. The following keywords were used in various combinations: DSWI, post-sternotomy complication, and sternal reconstruction. The following exclusion criteria were applied hierarchically: (1) not relevant to sternal reconstruction; (2) not relevant to post-surgical sternal complications; (3) not including feasible clinical applications or innovation; and (4) not in English. The abstracts were manually screened by four of the authors (LS, PC, EA, and PDS) separately and subsequently matched for accuracy.

\section{RESULTS}

Extensive variation was found in the studies concerning DSWI, to the point that it was impossible to compare the individual publications in a systematic manner. The identified articles were in fields ranging from cardio-thoracic surgery to infectious disease treatments, microbiology, wound healing, and surgical re-

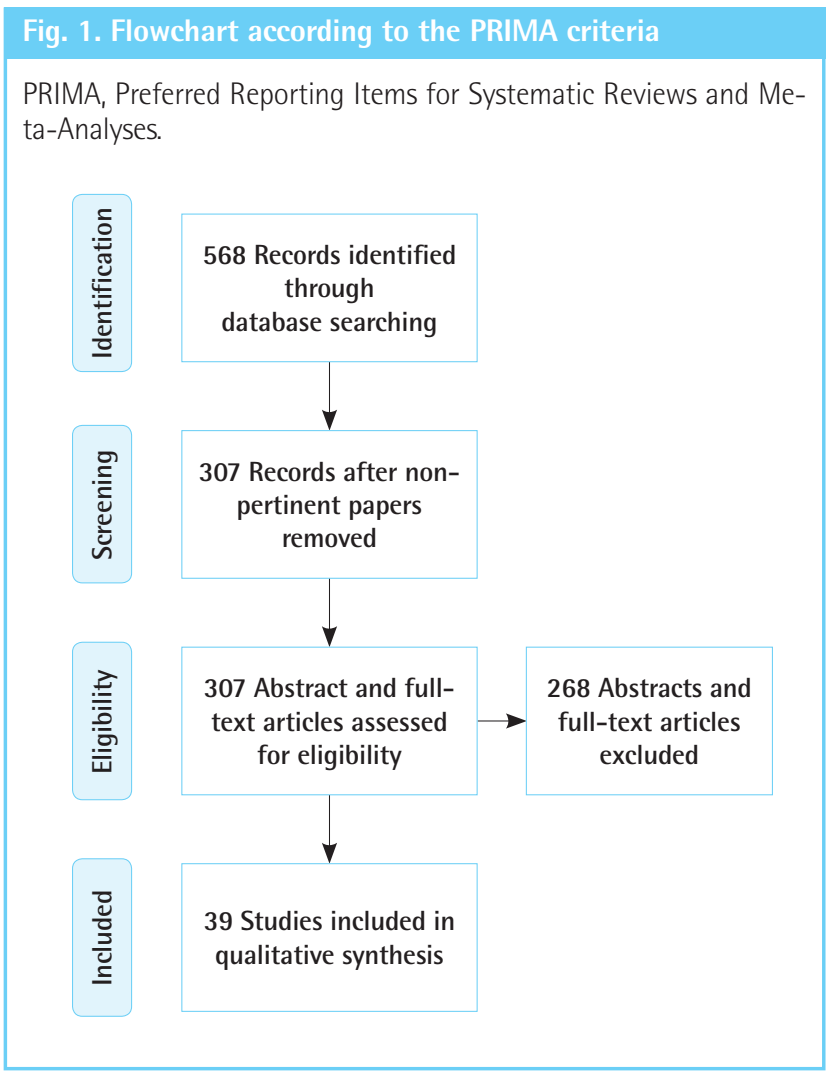

construction. Given these dramatic differences in terms of population, aims, and evaluated outcomes, it was not possible to perform a meta-analysis that would gather and combine the results. However, all publications were comprehensively analyzed, yielding an updated picture of the evidence base on the multidisciplinary treatment of DSWI, from prevention to final reconstruction.

\section{Classification of sternal complications}

Considering the multitude of possible presentations of wound infections post-sternotomy, the first step in our analysis was to extract proper definitions and classifications from the analyzed publications. DSWI can involve the skin, subcutaneous tissue, and eventually bone, with heterogeneous definitions in the literature [8]. The following classification, which was presented by Rupprecht and Schmid [9], addresses appropriate treatment according to clinical findings. This classification integrates the El Oakley and Wright classification [10], which is generally applied for sternal complications, and facilitates better planning of reconstructive options.

\section{Rupprecht and Schmid classification}

\section{Sternal instability without infection}

This condition develops through high traction forces to the sternal edges after surgery (e.g., severe coughing); such forces may damage osseous integrity and lead to early postoperative sternal instability. Alternatively, a more gradual gliding trauma can lead to cartilaginous metaplasia, usually resulting in chronic pain syndromes [9].

\section{DSWI without sternal instability}

Wound dehiscence without sternal instability is more likely to be seen in patients who, despite a good sternal structure, present with a considerable amount of tension on the skin and soft tissues, such as obese patients or women with breast hypertrophy. Similarly, patients with diabetes are generally predisposed to impaired wound healing, with on-going tension on the medial sternotomy line aggravating the problem, ultimately resulting in a deep wound infection [9].

DSWI with sternal instability

The combination of DSWI with sternal instability represents the most critical situation. Continuous movement (related to rupture or loosening of sternal wires) of the sternal edges leads to inflammation, effusion, and secondary infection. Frequently, due to extreme friability of the infected bone and cartilage, total destruction of the sternum may be present, and the wires can further induce bone fragmentation and eventually bone loss. 
Severe respiratory problems associated with pneumonia are generally common in these patients, further aggravating the clinical scenario [9]. Purulent mediastinitis with necrotic sternal bone can be considered the most advanced and dangerous stage of a DSWI.

Would complications can be further classified according to the timing of infection presentation [11]. Type I wounds occur within the first few days post-surgery, involve early wound separation with or without sternal instability, and are characterized by sero-sanguineous drainage in absence of cellulitis, costochondritis, or osteomyelitis. Type II wounds occur within the first few weeks and are characterized by purulent drainage, cellulitis, mediastinal suppuration, and positive cultures (eventually leading to fulminant mediastinitis). Type III wounds occur months to years after surgery and are distinguished by the presence of chronic draining sinus tracts, localized cellulitis, osteomyelitis, or retained foreign bodies (Table 1).

\section{Prevention of sternal complications}

The literature showed significant variation in the incidence of DSWI among different centers, ranging from $0.8 \%$ to $6 \%[5,6]$. Prevention played a crucial role in lowering this rate. Being aware of risk factors, properly selecting patients, and improving perioperative care were identified as critical preventive measures.

\section{Patient risk factors}

DSWI was found to be related to various predisposing elements. The following patient-related risk factors were cited in the literature: obesity, diabetes, coronary artery disease, low ejection fraction, steroid treatment, chronic infections, advanced age, active smoking, osteoporosis, end-stage renal disease, and chronic lung disease. Several procedure-related risk factors that significantly impair wound healing were identified, such as inadequate skin preparation, use of bone wax, emergency operation, non- skeletonized (pedicled) or bilateral harvesting of the internal mammary artery (IMA) [12], high-volume red blood cell transfusions, any platelet transfusion [13-15], prolonged operative time and perfusion time, sternal rewiring, postoperative bleeding, use of an intra-aortic balloon pump, extensive use of electrocautery, and late prophylactic antibiotic administration $(>60$ minutes prior to incision) $[16,17]$. Strong coughing, raising the intra-thoracic pressure up to $300 \mathrm{mmHg}$, was considered to be a factor with a considerable effect on sternal stability, as it induces strong shearing forces that may easily untwist the wires $[18,19]$.

\section{Procedure-related risk factors}

Coronary artery bypass graft (CABG) surgery using the bilateral internal mammary arteries (BIMA) instead of a single IMA (SIMA) increases the risk of DSWI [20], due to a dramatic decrease in sternal blood flow; this tendency is particularly noticeable with pedicle harvesting [21]. The Gatti score [22] can be used to identify high-risk patients, allowing a reasonable preoperative selection according to risk factors. Being aware of DSWI risk can help the surgeon choose between different CABG surgical techniques (skeletonized BIMA or SIMA), to adopt effective perioperative measures, and to implement closer postoperative wound follow-up.

\section{Perioperative care}

Various aspects of perioperative primary surgical care were reported to have a significant impact on DSWI risk in the examined literature.

In addition to meticulous disinfection and compliance with sterility principles, the topical use of antimicrobials applied to the sternum during cardiac procedures combined with standard intravenous agents may yield satisfactory results for DSWI prevention. Osawa et al. [23] showed that spraying a solution of gentamicin and cefazolin on the surgical site multiple times during cardiac surgery had beneficial effects in terms of protecting

\section{Table 1. Most commonly used classifications of postoperative sternal wound infection complications $[9,10]$}

\begin{tabular}{|c|c|c|c|c|}
\hline \multicolumn{5}{|c|}{ Classification } \\
\hline & Timing of infection & Clinical findings & & Timing of infection and risk factors \\
\hline & Pairolero & Rupprecht and Schmid & & El Oakley \\
\hline Type I & In the first week & Noninfected sternal instability & Type I & DSWI presenting within 2 weeks after operation in the absence of risk factors \\
\hline Type II & Between 2 and 6 weeks & DSWI without sternal instability & Type ॥ & DSWI presenting at 2-6 weeks after operation in the absence of risk factors \\
\hline \multirow[t]{5}{*}{ Type III } & After 6 weeks to years & DSWI with sternal instability & Type Illa & DSWI like type I in the presence of one or more risk factors \\
\hline & & & Type IIIb & DSWI like type II in the presence of one or more risk factors \\
\hline & & & Type IVa & DSWI like type I, II, or III after one failed therapeutic trial \\
\hline & & & Type IVb & DSWI like type I, II, or III after more than one failed therapeutic trial \\
\hline & & & Type V & DSWI presenting for the first time $>6$ weeks after operation \\
\hline
\end{tabular}


high-risk patients (patients with diabetes and those undergoing emergency cardiac procedures). Prophylactic perioperative antibiotic therapy and intra-nasal prophylaxis with mupirocin are also effective in reducing the incidence of postoperative sternal wound infection [24].

Sternal fixation techniques were found to significantly affect the infection rate. Moving beyond traditional sternal closure with sternal wires, hybrid techniques have been proposed to achieve better sternal stability [2]. Combining stainless steel monofilament wire sutures with peristernal cable-tie devices (ZipFix; DePuy Synthes GmbH, Oberdorf, Switzerland) appeared to be an effective way of reducing the DSWI rate.

Dehiscence and infection of the sternum may preclude rewiring, especially in patients with multiple morbidities [25]. Titanium plate sternal fixation, despite making it more difficult to quickly re-access the mediastinum, reduced the need for multiple rounds of debridement, offering good sternal stability and reducing mortality (11.1\%) when compared to traditional wire re-fixation (19.2\%) [26]. This strategy was suggested for primary surgery in high-risk patients or in patients undergoing sternal wound debridement.

Finally, autologous platelet-rich plasma (PRP) use for the pre- vention of DSWI has been proposed recently, with good clinical results [27]. During the inflammatory phase of wound healing, PRP stimulates the release of growth factors such vascular endothelial growth factor and transforming growth factor beta, which are believed to stimulate cell proliferation, migration, differentiation, and matrix synthesis. These same growth factors can positively affect chondrocyte metabolism, increase chondrogenesis, and improve bone healing and regeneration [27]. PRP wound irrigation was found to be safe and cost-effective, improving healing and leading to a lower incidence of sternal infections. Moreover, PRP was able to inhibit the proliferation of Staphylococcus aureus, one of the most common bacteria responsible for DSWI [27].

\section{Debridement and acute treatment of sternal complications}

Despite the heterogeneity of the investigated literature, debridement was a common element_-as expected_of all treatment strategies for DSWI. After analyzing articles proposing treatment algorithms for debridement, we were able to match the appropriate treatment to specific clinical situations, as shown in the practical algorithm in Fig. 2.

\section{Fig. 2. DSWI acute treatment algorithm}

Acute treatment and surgical debridement algorithm for postoperative sternal wound infection complications. DSWI, deep sternal wound infection.

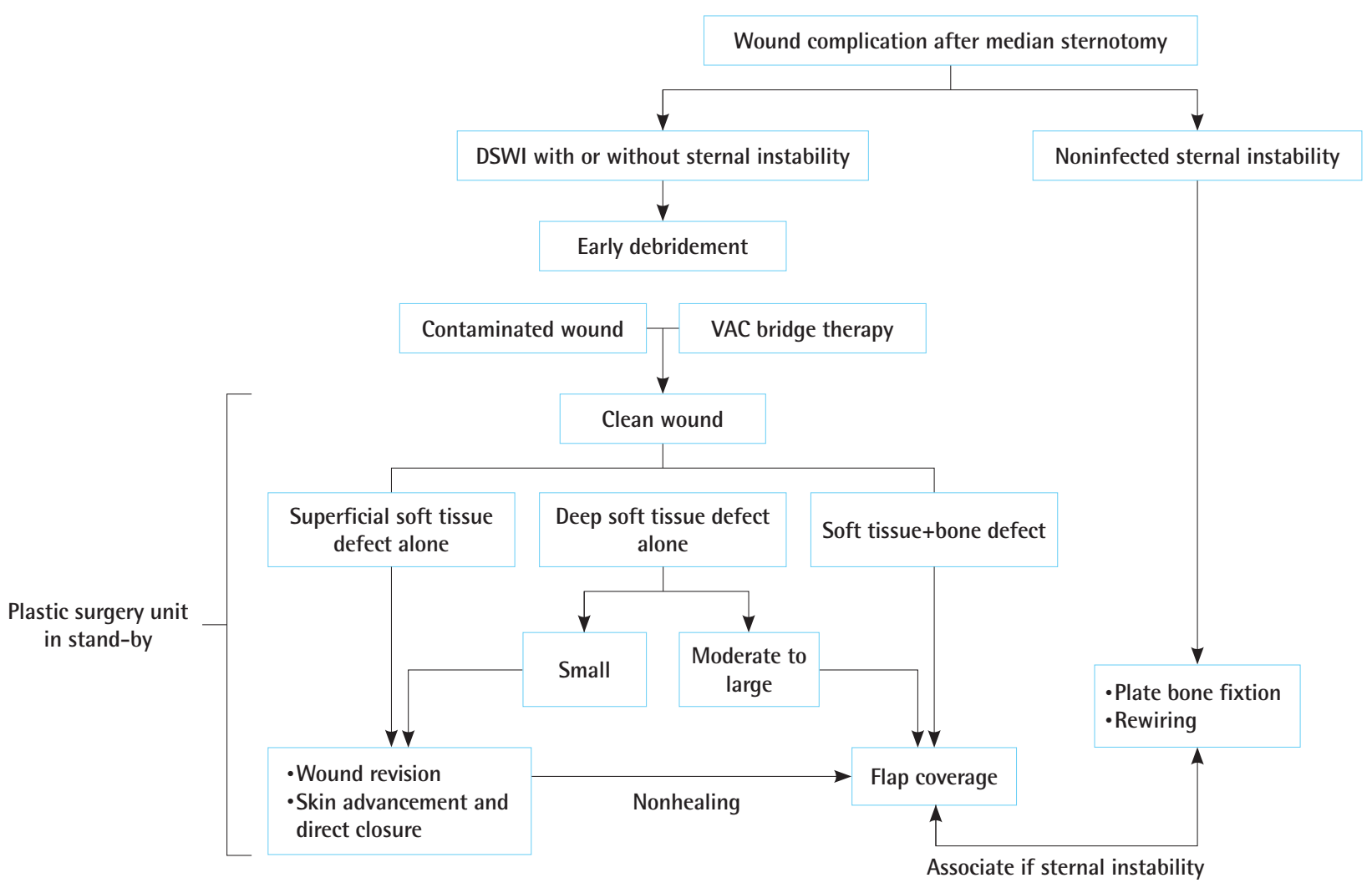




\section{Noninfected sternal instability}

The surgical treatment of noninfected sternal instability depends on the integrity of the sternal wound edges, and the bone quality is crucial for achieving a good result. In cases of simple wire loosening, authors generally suggested rewiring or, occasionally, simple strengthening. However, in nearly half of the cases [26], this approach was found to be ineffective, requiring further re-exploration with an increased risk of a secondary deep infection [25]. In a recent work, Wang and Wang [26] demonstrated the superiority of plate fixation in cases of sternal instability. Using three to four transverse titanium plates together with one $\mathrm{H}$-shaped short plate after bone reduction, the authors achieved proper sternal fixation, reducing the need for further surgery. No differences were found between the alternative types of sternal fixation [28-31]. All these techniques served their intended purpose well, but were not found to be superior to standard trauma plates, which can be used in most instances [32].

\section{DSWI without sternal instability}

In cases of DSWI without sternal instability, in order to eliminate the infectious focus, extensive debridement and aggressive antibiotic treatment are mandatory. Surgical washout and debridement should progress until adequately perfused tissue is visible. If the sternum is stable enough, any infected sternal wires should be ablated to eliminate the bacterial biofilm, and the entry sites of the wires into the sternal bone should be inspected and debrided [9].

While the basic principles of wound debridement were commonly practiced and widely accepted in the analyzed publications, wound closure was more controversial. Although early closure seemed to reduce secondary wound contamination and infection [25], it has also been proposed that in cases of extensive infection, secondary closure of the wound after an appropriate open treatment period and repeated surgical looks would be preferable [33]. Bridge treatment with negative-pressure therapy (VAC) until the wound becomes clean and granulation tissue appears was generally seen as an attractive alternative $[34,35]$. VAC therapy has been shown to increase parasternal blood flow, even in the face of IMA harvest, and to reduce the initial bacterial load [36]. According to the recent literature, the sternal bone can heal despite the presence of a low bacterial load. Prolonged use of VAC garments may promote the presence of this chronic bacterial load, implying that the duration of VAC bridge therapy should be shortened in order to lower the risk of secondary and polymicrobial wound infection [25].

\section{DSWI with sternal instability}

Unfortunately, the most common sternal complication after car- diac surgery is partial or complete dehiscence, resulting in fragmentation of the sternal structure with a concomitant wound infection. In contrast, it is very unlikely for a superficial infection to affect an initially stable sternum to the point that it has to be un-joined due to extensive infectious involvement of the sternal wires [9]. Debridement of both the wound and sternum (partial or total according to the specific clinical scenario) was mentioned in all analyzed articles as a step of the foremost importance. The main debate was whether primary closure should be performed when a "clean" mediastinum is obtained. Continuous antibiotic irrigation with rewiring of the sternum and closure of the wound is a consolidated treatment that was devised in 1963 [37,38]. However, since the efficacy of mediastinal rinsing is limited, a considerable risk of an infectious relapse, with a consequent need for wound revision, remains [39]. Leaving the mediastinum open is currently considered a more acceptable alternative. In cases of purulent mediastinitis, after extensive debridement and multiple rinsing, the mediastinum is packed with a VAC system, which helps to resolve the infection effectively [40].

Among methods of secondary wound closure after mediastinum decontamination, the omentum was the preferred choice in cases of massive bone defects or defects covering the entire mediastinum and the exposed pericardium [41]. Other flaps have been described as suitable options depending on the defect size and volume, location, and available vascular axis (Figs. 3, 4), as discussed below. As mentioned before, rewiring can be difficult due to sternal fragility; therefore, placement of titanium plates is associated with a better outcome [26].

\section{Antimicrobial treatment}

According to the analyzed papers, S. aureus was the most commonly involved pathogen [25], although a significant increase in the incidence of DSWI due to slower-growing pathogens such as coagulase-negative staphylococci (CoNS) and certain fungi has been observed since the late 1990s [42-44]. Recent studies found CoNS to be the most common pathogen in cases of DSWI, with rates from $40 \%$ to $62 \%$ [45-47]. Candidal mediastinitis after cardiac surgery used to be exceedingly rare [48]; however, an incidence of $20 \%$ has been recently reported [49]. The tendency of CoNS and Candida to form a biofilm in deeper structures, despite conventional antifungal and antibiotic therapy [50], can result in a mutualistic inter-species relationship that leads to combined vancomycin and fluconazole resistance [51].

Secondary pathogens generally belong to a heterogeneous group, mostly involving gram-negative bacilli such as Escherichia coli and Klebsiella. Less common described pathogens were enterococci and streptococci, non-fermentative bacilli, and fungi, 


\section{Fig. 3. Clinical presentation of a DSWI}

(A) Post-sternotomy wound infection. The wound involved skin and deep soft tissues, including bone with chronic osteomyelitis. Dehiscence appeared 3 months after the last surgical procedure (classified as Pairolero type III and El Oakley type V) [10,11]. (B) Intraoperative debridement. The defect partially involved the sternum, including the sternal notch. (C) Intraoperative phase of flap coverage surgery. The presence of one spared internal mammary artery, the width of the wound, and the necessity of reaching the sternal notch led the surgeon to choose a vertical rectus abdominis musculocutaneous flap based on the contralateral superior epigastric artery. (D) Surgical results at 3 weeks postoperatively. The patient fully recovered, with full working activity and no infectious complications at 2 years of follow-up.
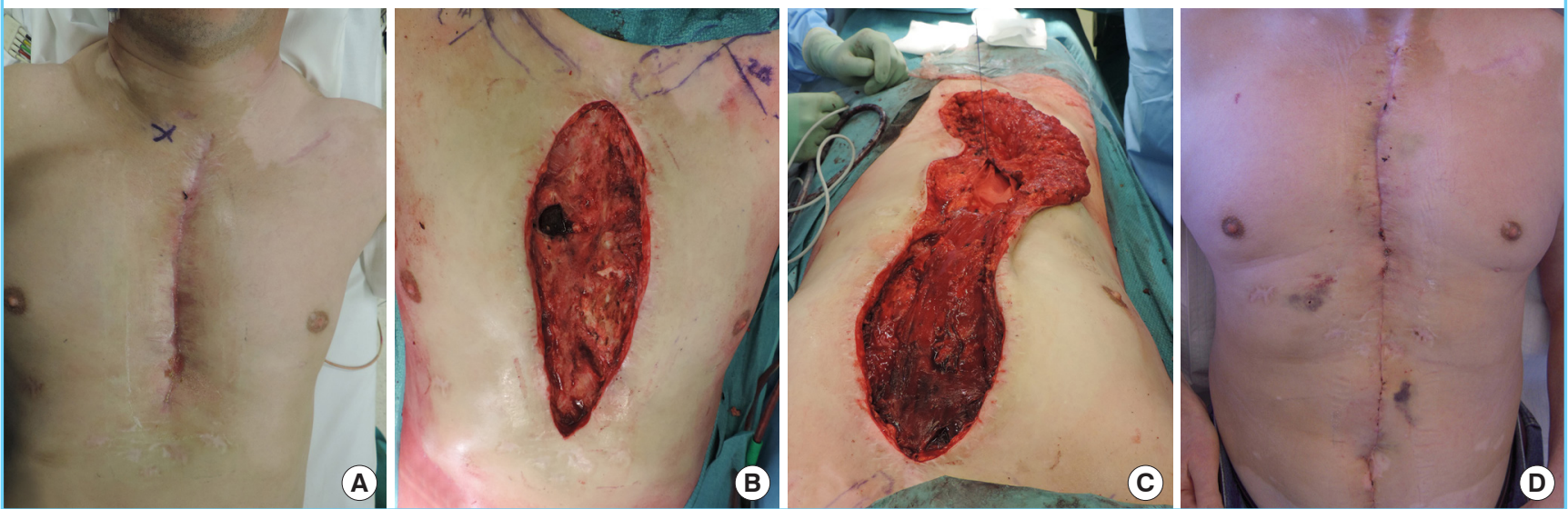

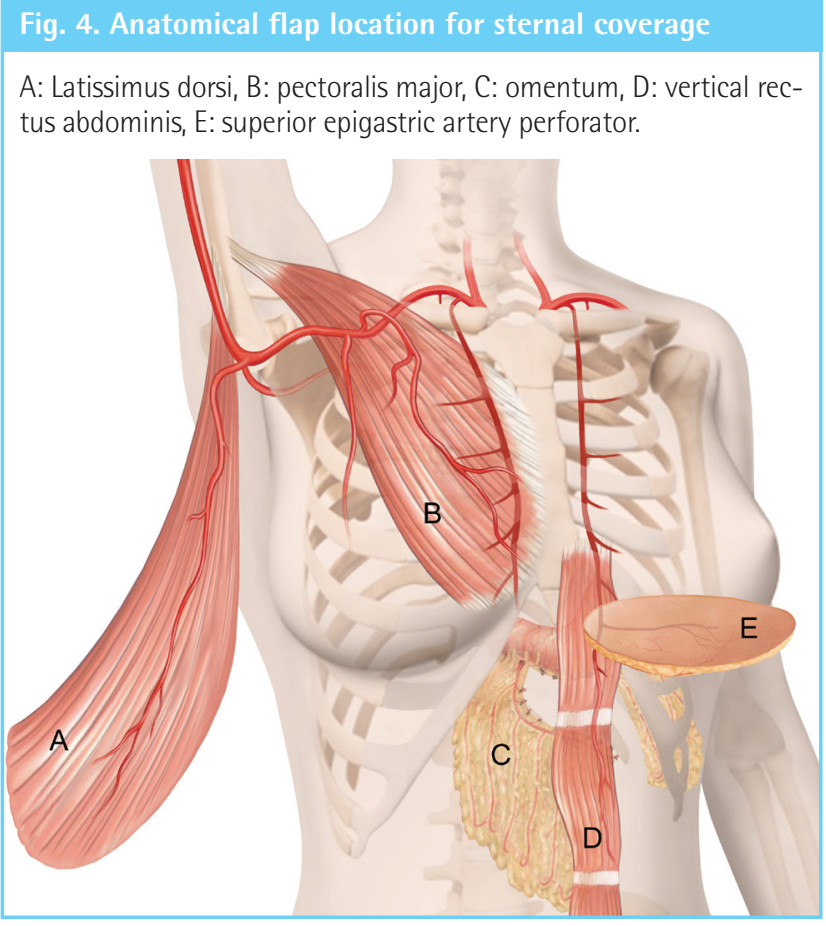

in order of frequency $[25,40]$.

The authors agreed on the fact that initial empiric antibiotic therapy must include broad coverage against methicillin-resistant gram-positive, gram-negative, and anaerobic organisms. Vancomycin represents the first choice in the majority of cases, followed by oral therapy based on rifampicin, quinolone, and cotrimoxazole. According to the literature, culture-directed therapy should be started as soon as a microbiological analysis is available, and systemic intravenous antibiotics should be maintained for at least 6 weeks. Antifungal therapy should be administered in the absence of clinical improvement on antibiotics [25].

\section{Surgical reconstruction}

The analyzed articles agreed that the best results are achieved with a multidisciplinary approach, in which plastic surgeons are alerted when a wound complication after sternotomy is suspected. Patients who were referred to plastic surgery specialists were at a significantly lower risk of ventilator dependence, development of pressure sores, prolonged length of hospital stay, and mortality than patients who were initially managed conservatively [52]. Brandt and Alvarez [41] compared muscle or omental flap reconstruction to traditional treatment involving debridement and closed drainage. They demonstrated a $22 \%$ rate of major complications and a $0 \%$ mortality rate in the flap group, compared with a $92 \%$ rate of major complications and a 33\% mortality rate in the traditionally treated patients. Muscle flaps promote early wound closure and reduce mortality. In a review of 211 sternal infections treated with muscle flaps, successful wound closure occurred in $95 \%$ of patients, with a mortality rate of $5.7 \%$ [53]. Muscle flaps and omentum flaps were effective at expediting the resolution of infections in the most frightening cases of DSWI, and may be the only viable option in cases of unresponsive infections, bleeding, and large chest-wall defects [54].

In the sternal region, the workhorse procedures of reconstructive surgery include the unilateral or bilateral pectoralis major flap, pectoralis major turn-over flap, latissimus dorsi flap, rectus abdominis flap, and greater omentum flap (Fig. 4). These flaps are described below with their specific advantages, disadvantag- 


\section{Fig. 5. Proposed reconstruction algorithm according to the literature}

IMA, internal mammary artery; VRAM, vertical rectus abdominis musculocutaneous.

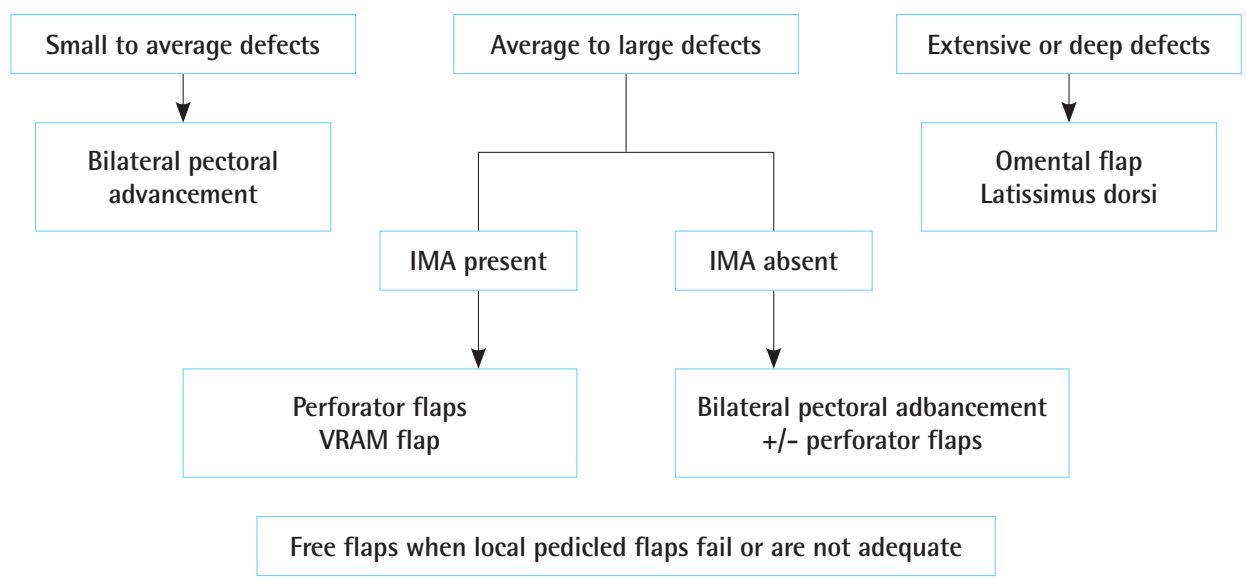

es, and indications as found in the literature. If the muscles mentioned above fail to succeed, or are simply unavailable or contraindicated, microsurgical free flaps can be an effective reconstructive possibility, as shown in the reconstruction algorithm in Fig. $5[55,56]$.

\section{Pectoralis major flap}

The most used flap in sternal wound reconstruction is the pedicled pectoralis major muscle flap based on the thoracoacromial artery. Back-cutting the superior medial segment of the pectoralis muscle maintains its blood supply and allows for more caudal coverage. To guarantee full sternal coverage and support to the xyphoid, the pectoralis is then approximated to the cephalad portion of the rectus sheath to help with full sternal coverage and support. Bilateral pectoralis major muscle flaps have the key advantage of not being dependent on the IMA [57]. Moreover, they do not add a new incision, do not compromise the function and appearance of the chest, and do not affect the activity of the upper limb. Another key advantage is the possibility of harvesting the flap on the medial perforators coming from the IMA only; this often allows a combination of a turnover pectoralis flap on the side from which the IMA has not been harvested and a unipedicled rotational advancement flap on the ipsilateral side. The pectoralis major is more suitable for small to moderate defects, as long as the key zone (mid-chest) is covered by the peripheral part of the flap. Similarly, cachectic patients with weak pectoralis major muscles should be considered at potential risk of flap failure or insufficient coverage.

\section{Vertical rectus abdominis musculocutaneous flap}

The rectus abdominis flap is pedicled on the superior epigastric artery and is advocated due to its ease of dissection and its wide arc of rotation, which allows it to reach the sternal notch as well as the most common site of breakdown, the lower third of the sternal wound. Although hernia formation or protrusion is a concern, Davison et al. [58] significantly diminished this complication, from a reported $50 \%$ in some studies to $2 \%$ in their series, by leaving the rectus fascia in place and closing in two layers. When comparing rectus abdominis flaps alone and the modified pectoralis major flap with anterior rectus sheath extension, the rectus group showed a statistically significant improvement in terms of preventing dehiscence of the inferior third of the sternum [58]. In our experience, this flap allows for fast harvesting, minimal bleeding, and efficient coverage. In moderate to large defects including the sternal bone, it helps to cover the debrided sternum and to fill bone defects. A major drawback is that it cannot be used when both IMAs have been harvested. Elastic abdominal belt compression is advised for 6 to 12 weeks postoperatively, according to the patient's work activities.

\section{Omental flap}

The omental flap is a useful reconstructive option because it can conform to the deepest recesses of the sternal wound and has noteworthy immunological properties, showing superiority when compared to the pectoralis flap in preventing sepsis-related morbidity [59]. The omental flap requires an additional surgical site, but the minimally invasive laparoscopic approach reduces the risks related to this procedure. Sternal wound defect coverage by the omentum has often been considered as a secondary reconstructive option when pectoralis or rectus abdominis flaps have failed or are not available [60]. A recent report by Stump et al. [61] suggested that patients with diabetes treated 
with omental transposition showed a decreased need for flap revision compared to those who received a pectoralis muscle flap. In our experience, the main indications for this flap are large defects or massive sternal debridement and mediastinitis.

\section{Perforator flaps}

Perforator flaps represent a totally new concept since their introduction in reconstructive surgery. Instead of relying on the angiosome concept, the "perforasomes" nourished by perforator vessels allow minimally invasive flap harvesting, sparing the main vessel axis (e.g., the IMA) and functional muscle tissues [62]. Superior epigastric artery perforator (SEAP) flaps, instead of traditional musculocutaneous flaps, have become a common choice when moderate to large defects are present $[63,64]$. After detecting the emergence of the most suitable perforator using a handheld acoustic Doppler (located next to the sternal origin of the rectus abdominis), the axis of the flap is drawn horizontally, following the skin perforator vascularization of the trunk reported by Saint-Cyr and colleagues $[62,64]$. If necessary, in order to reach more distal wound edges, the perforator artery and veins can be dissected under loupe magnification through the deep fascia until it becomes possible to rotate the flap without twisting the pedicle. Considering the midline defect, this is usually not necessary, and a $90^{\circ}$ to $100^{\circ}$ rotation into the defect (Fig. 5) can be easily achieved, even without completely isolating the skin paddle, leaving a skin bridge to help venous drainage and to avoid venous congestion. Propeller flaps based on superior epigastric perforators are a fast, reliable technique with minimal donor site morbidity; they are suitable for use in patients who require coverage of at least the lower-two thirds of the sternum, which is the usual site of DSWI [64]. In our experience, SEAP flaps can be occasionally harvested in patients without an available IMA, as the flap is still vascularized by the inferior epigastric artery. In these particular cases, computed tomography angiography is generally mandatory to obtain information on perforator quality and size. Another major advantage is minimal donor site morbidity, as the scar is hidden in the inframammary fold. Since flaps over $25 \mathrm{~cm}$ in length may cause distal skin ischemia [65], we believe that a combination with a unilateral pectoralis major flap is reasonable for longitudinally long defects. The disadvantages of the SEAP flap include the potential need of the IMA axis and its limited length.

The IMA perforator (IMAP) may be more adequate from the notch defect up to the xiphoid, with relatively minimal dissection and morbidity compared with conventional flaps. Although IMAP flaps are generally harvested as fasciocutaneous flaps, they can be harvested as musculocutaneous flaps, including part of the pectoralis major for deeper defects [66]. IMAP flaps rely on second or third intercostal perforators that are generally spared despite the use of the IMA more distally when harvesting for a CABG. However, similarly to the SEAP flap after bilateral IMA use for cardiac procedures, preoperative computed tomography angiography is strongly suggested for operative planning. The main disadvantage of this flap is a more difficult perforator dissection when compared to the SEAP and the resulting subclavicular scar, which is aesthetically displeasing in women.

Perforator flaps are an important tool for reconstructive surgeons. Their relative noninvasiveness can be particularly useful in fragile patients in whom extensive procedures should be avoided.

\section{DISCUSSION}

DSWI can lead to serious complications if not treated aggressively, making prevention and early management important. The optimal treatment is still controversial, and it was impossible to combine the results of the different papers analyzed herein to obtain cumulative evidence of the treatment to be chosen. However, the heterogeneity of the analyzed publications is in line with the multidisciplinarity and diverse aspects of DSWI. Although a systematic review of the "best treatments" could not be performed, smaller groups of papers relating to different aspects of DSWI (patient- and procedure-related risk factors, perioperative care and fixation, type and timing of debridement, antibiotic treatment, and types of surgical reconstruction) were reviewed to generate a comprehensive picture of the evidence available for the complex management of DSWI. Through this review, we developed treatment algorithms for both debridement and reconstruction. As a general rule, when dealing with DSWI, both the instability and infection must be treated, due to the severe systemic repercussions of both factors. Surgical wound and partial or total sternal bone debridement is a priority. Mediastinal inspection and cleaning, including excision of necrotic tissue, fragment removal, and extensive rinsing, are necessary when reopening the sternum. The sternal edges must be cleared from syndesmotic coverage.

In our experience, flap coverage should not be delayed in cases of chronic DSWI (especially post-transplant or after implantation of Dacron grafts) after VAC therapy ( $>2$ weeks). Greig et al. [67] proposed an anatomical classification based on the site of infection. They recommended pectoralis major muscle flaps for defects in the upper half of the sternum, and combined pectoralis major and rectus abdominis muscle flaps for defects in the lower half and the whole sternum. Another algorithm (the Cologne-Merheim approach) [68] presents a strategy based on wound size and depth, in which small wounds (up to $6 \mathrm{~cm}$ ) are 
to be covered by unilateral or bilateral musculocutaneous pectoralis flaps. Unilateral pedicled pectoralis flaps are used for medium wounds (between 7 and $12 \mathrm{~cm}$ ). For large wounds (>13 $\mathrm{cm})$, a left pedicled latissimus dorsi flap is advised.

In our opinion, the flap choice depends on wound dimensions, deep tissue involvement, anatomical findings (presence or absence of IMA), and microsurgical skills (Fig. 1). If the defect is relatively small, bilateral pectoral advancement should be the first treatment option. In cases of larger defects, the presence of at least one IMA should orient the surgeon to choose between a perforator flap or a vertical rectus abdominis musculocutaneous flap, according to the need for muscular coverage, the necessity of covering the sternal notch, and the patient's general status. If the IMA is absent, a bilateral pectoral advancement flap or perforator flap (after appropriate perforator confirmation by computed tomography angiography) can be used. An omental flap should be considered for extensive or deep defects, unstable patients, and patients with a high risk of infection [9]. As an alternative to the omentum, among pedicled flaps, we have found the latissimus dorsi flap to be a viable solution in cases of massive sternal or parasternal defects. When meticulously dissected down to its pedicle (thoracodorsal artery), good coverage of the midline can be achieved.

Microsurgical and free flaps should be considered when local pedicled flaps have failed or seem inadequate. Nourishing arteries and veins for free flaps can be often found in the operative field by experienced surgeons. Arteriovenous loops have been described as an alternative if the recipient vessels are depleted [56]. However, considering the risk of thrombosis at the anastomotic sites, we believe that microsurgical reconstructions should be performed after accurate planning, confirmation of patient hemodynamic stability (e.g., discontinuation of vasopressors), and resolution of the acute infectious inflammatory phase.

Patients should be referred early to plastic surgeons, during the acute or post-acute infectious phase. This allows proper surgical planning in order to obtain the best possible reconstructive outcomes, avoiding further infectious complications that can be life-threatening in the mediastinal setting.

\section{CONCLUSION}

DSWI is a potentially life-threatening complication of cardiac surgery. Despite the low incidence, DSWI-related morbidity, mortality, and costs are extremely high. Prevention, aggressive acute treatment, and early involvement of the reconstructive team are crucial steps to prevent and treat DSWI-related complications.
In the operative flowchart presented herein, we included all modifiable aspects of the care process, showing that a standardized clinical protocol can lower DSWI incidence and morbidity.

\section{NOTES}

\section{Conflict of interest}

No potential conflict of interest relevant to this article was reported.

\section{Ethical approval}

The study was approved by the Lausanne University Hospital (CHUV) Review Board and performed in accordance with the principles of the Declaration of Helsinki. Written consent were obtained.

\section{Patient consent}

The patients provided written informed consent for the publication and the use of their images.

\section{Author contribution}

Conceptualization: Jabbour G. Data curation: di Summa PG, Schiraldi L. Formal analysis: Giordano S, di Summa PG. Acquisition: Schiraldi L, Jabbour G, Abdelnour E. Methodology: Raffoul W, Centofanti P, Gonzalez M. Project administration: di Summa PG. Visualisation: Schiraldi L, Jabbour G, Giordano S. Writing-original draft: Schiraldi L, Jabbour G. Writing- review: di Summa PG, Gonzalez M, Raffoul W.

\section{ORCID}

Luigi Schiraldi https://orcid.org/0000-0002-9766-5185

Salvatore Giordano https://orcid.org/0000-0001-5981-6870

Etienne Abdelnour https://orcid.org/0000-0003-4002-9046

Michel Gonzalez https://orcid.org/0000-0001-8705-4279

Wassim Raffoul https://orcid.org/0000-0002-3524-0077

Pietro Giovanni di Summa https://orcid.org/0000-0002-

$1431-4479$

\section{REFERENCES}

1. McGregor WE, Trumble DR, Magovern JA. Mechanical analysis of midline sternotomy wound closure. J Thorac Cardiovasc Surg 1999;117:1144-50.

2. Stelly MM, Rodning CB, Stelly TC. Reduction in deep sternal wound infection with use of a peristernal cable-tie closure system: a retrospective case series. J Cardiothorac Surg 2015;10:166.

3. Cayci C, Russo M, Cheema FH, et al. Risk analysis of deep 
sternal wound infections and their impact on long-term survival: a propensity analysis. Ann Plast Surg 2008;61:294301.

4. Sjogren J, Malmsjo M, Gustafsson R, et al. Poststernotomy mediastinitis: a review of conventional surgical treatments, vacuum-assisted closure therapy and presentation of the Lund University Hospital mediastinitis algorithm. Eur J Cardiothorac Surg 2006;30:898-905.

5. Tang AT, Ohri SK, Haw MP. Novel application of vacuum assisted closure technique to the treatment of sternotomy wound infection. Eur J Cardiothorac Surg 2000;17:482-4.

6. Eklund AM, Lyytikainen O, Klemets P, et al. Mediastinitis after more than 10,000 cardiac surgical procedures. Ann Thorac Surg 2006;82:1784-9.

7. Moher D, Altman DG, Liberati A, et al. PRISMA statement. Epidemiology 2011;22:128.

8. van Wingerden JJ, de Mol BA, van der Horst CM. Defining post-sternotomy mediastinitis for clinical evidence-based studies. Asian Cardiovasc Thorac Ann 2016;24:355-63.

9. Rupprecht L, Schmid C. Deep sternal wound complications: an overview of old and new therapeutic options. Open J Cardiovasc Surg 2013;6:9-19.

10. El Oakley RM, Wright JE. Postoperative mediastinitis: classification and management. Ann Thorac Surg 1996;61: 1030-6.

11. Pairolero PC, Arnold PG. Management of recalcitrant median sternotomy wounds. J Thorac Cardiovasc Surg 1984;88: $357-64$.

12. Centofanti P, Savia F, La Torre M, et al. A prospective study of prevalence of 60-days postoperative wound infections after cardiac surgery: an updated risk factor analysis. J Cardiovasc Surg (Torino) 2007;48:641-6.

13. Ridderstolpe L, Gill H, Granfeldt H, et al. Superficial and deep sternal wound complications: incidence, risk factors and mortality. Eur J Cardiothorac Surg 2001;20:1168-75.

14. Robinson PJ, Billah B, Leder K, et al. Factors associated with deep sternal wound infection and haemorrhage following cardiac surgery in Victoria. Interact Cardiovasc Thorac Surg 2007;6:167-71.

15. Cutrell JB, Barros N, McBroom M, et al. Risk factors for deep sternal wound infection after cardiac surgery: influence of red blood cell transfusions and chronic infection. Am J Infect Control 2016;44:1302-9.

16. Nishida H, Grooters RK, Soltanzadeh H, et al. Discriminate use of electrocautery on the median sternotomy incision: a $0.16 \%$ wound infection rate. J Thorac Cardiovasc Surg 1991;101:488-94.

17. Tang GH, Maganti M, Weisel RD, et al. Prevention and management of deep sternal wound infection. Semin Thorac Cardiovasc Surg 2004;16:62-9.

18. Irwin RS. Complications of cough: ACCP evidence-based clinical practice guidelines. Chest 2006;129(1 Suppl):54S$58 \mathrm{~S}$.

19. Casha AR, Yang L, Kay PH, et al. A biomechanical study of median sternotomy closure techniques. Eur J Cardiothorac Surg 1999; 15:365-9.

20. Gatti G, Dell'Angela L, Barbati G, et al. A predictive scoring system for deep sternal wound infection after bilateral internal thoracic artery grafting. Eur J Cardiothorac Surg 2016; 49:910-7.

21. Calafiore AM, Weltert L, Mauro MD, et al. Internal mammary artery. Multimed Man Cardiothorac Surg 2005;2005: mmcts.2004.001008.

22. Gatti G, Barbati G, Luzzati R, et al. Prospective validation of a predictive scoring system for deep sternal wound infection after routine bilateral internal thoracic artery grafting. Interact Cardiovasc Thorac Surg 2016;22:606-11.

23. Osawa H, Yoshii S, Abraham SJ, et al. Topical spraying of cefazolin and gentamicin reduces deep sternal wound infections after heart surgery: a multicenter, large volume, retrospective study. Gen Thorac Cardiovasc Surg 2016;64:197202.

24. Ennker IC, Pietrowski D, Vohringer L, et al. Surgical debridement, vacuum therapy and pectoralis plasty in poststernotomy mediastinitis. J Plast Reconstr Aesthet Surg 2009;62: 1479-83.

25. Chan M, Yusuf E, Giulieri S, et al. A retrospective study of deep sternal wound infections: clinical and microbiological characteristics, treatment, and risk factors for complications. Diagn Microbiol Infect Dis 2016;84:261-5.

26. Wang W, Wang S. Titanium plate fixation versus conventional approach in the treatment of deep sternal wound infection.J Cardiothorac Surg 2016;11:46.

27. Patel AN, Selzman CH, Kumpati GS, et al. Evaluation of autologous platelet rich plasma for cardiac surgery: outcome analysis of 2000 patients. J Cardiothorac Surg 2016;11:62.

28. Centofanti P, La Torre M, Barbato L, et al. Sternal closure using semirigid fixation with thermoreactive clips. Ann Thorac Surg 2002;74:943-5.

29. Huh J, Bakaeen F, Chu D, et al. Transverse sternal plating in secondary sternal reconstruction. J Thorac Cardiovasc Surg 2008;136:1476-80.

30. Coonar AS, Qureshi N, Smith I, et al. A novel titanium rib bridge system for chest wall reconstruction. Ann Thorac Surg 2009;87:e46-8.

31. Chou SS, Sena MJ, Wong MS. Use of SternaLock plating 
system in acute treatment of unstable traumatic sternal fractures. Ann Thorac Surg 2011;91:597-9.

32. Harston A, Roberts C. Fixation of sternal fractures: a systematic review.J Trauma 2011;71:1875-9.

33. Voss B, Bauernschmitt R, Will A, et al. Sternal reconstruction with titanium plates in complicated sternal dehiscence. Eur J Cardiothorac Surg 2008;34:139-45.

34. Morykwas MJ, Argenta LC, Shelton-Brown EI, et al. Vacuum-assisted closure: a new method for wound control and treatment: animal studies and basic foundation. Ann Plast Surg 1997;38:553-62.

35. Obdeijn MC, de Lange MY, Lichtendahl DH, et al. Vacuumassisted closure in the treatment of poststernotomy mediastinitis. Ann Thorac Surg 1999;68:2358-60.

36. Singh K, Anderson E, Harper JG. Overview and management of sternal wound infection. Semin Plast Surg 2011;25: 25-33.

37. Shumacker HB Jr, Mandelbaum I. Continuous antibiotic irrigation in the treatment of infection. Arch Surg 1963;86: 384-7.

38. Bryant LR, Spencer FC, Trinkle JK. Treatment of median sternotomy infection by mediastinal irrigation with an antibiotic solution. Ann Surg 1969;169:914-20.

39. Schimmer C, Reents W, Elert O. Primary closure of median sternotomy: a survey of all German surgical heart centers and a review of the literature concerning sternal closure technique. Thorac Cardiovasc Surg 2006;54:408-13.

40. Morisaki A, Hosono M, Murakami T, et al. Effect of negative pressure wound therapy followed by tissue flaps for deep sternal wound infection after cardiovascular surgery: propensity score matching analysis. Interact Cardiovasc Thorac Surg 2016;23:397-402.

41. Brandt C, Alvarez JM. First-line treatment of deep sternal infection by a plastic surgical approach: superior results compared with conventional cardiac surgical orthodoxy. Plast Reconstr Surg 2002;109:2231-7.

42. Gårdlund B, Bitkover CY, Vaage J. Postoperative mediastinitis in cardiac surgery: microbiology and pathogenesis. Eur J Cardiothorac Surg 2002;21:825-30.

43. Leung Wai Sang S, Chaturvedi R, Alam A, et al. Preoperative hospital length of stay as a modifiable risk factor for mediastinitis after cardiac surgery. J Cardiothorac Surg 2013;8:45.

44. Heilmann C, Stahl R, Schneider C, et al. Wound complications after median sternotomy: a single-centre study. Interact Cardiovasc Thorac Surg 2013;16:643-8.

45. Baillot R, Cloutier D, Montalin L, et al. Impact of deep sternal wound infection management with vacuum-assisted closure therapy followed by sternal osteosynthesis: a 15-year review of 23,499 sternotomies. Eur J Cardiothorac Surg 2010;37:880-7.

46. Matros E, Aranki SF, Bayer LR, et al. Reduction in incidence of deep sternal wound infections: random or real? J Thorac Cardiovasc Surg 2010;139:680-5.

47. Steingrimsson S, Gottfredsson M, Gudmundsdottir I, et al. Negative-pressure wound therapy for deep sternal wound infections reduces the rate of surgical interventions for early re-infections. Interact Cardiovasc Thorac Surg 2012;15: 406-10.

48. Clancy CJ, Nguyen MH, Morris AJ. Candidal mediastinitis: an emerging clinical entity. Clin Infect Dis 1997;25:608-13.

49. Jones G, Jurkiewicz MJ, Bostwick J, et al. Management of the infected median sternotomy wound with muscle flaps. The Emory 20-year experience. Ann Surg 1997;225:766-76.

50. Jabra-Rizk MA, Falkler WA, Meiller TF. Fungal biofilms and drug resistance. Emerg Infect Dis 2004;10:14-9.

51. Adam B, Baillie GS, Douglas LJ. Mixed species biofilms of Candida albicans and Staphylococcus epidermidis. J Med Microbiol 2002;51:344-9.

52. Cabbabe EB, Cabbabe SW. Immediate versus delayed onestage sternal débridement and pectoralis muscle flap reconstruction of deep sternal wound infections. Plast Reconstr Surg 2009;123:1490-4.

53. Rand RP, Cochran RP, Aziz S, et al. Prospective trial of catheter irrigation and muscle flaps for sternal wound infection. Ann Thorac Surg 1998;65:1046-9.

54. Kutsal A, Ibrisim E, Catav Z, et al. Mediastinitis after open heart surgery. Analysis of risk factors and management. J Cardiovasc Surg (Torino) 1991;32:38-41.

55. Luan A, Galvez MG, Lee GK. Flow-through omental flap to free anterolateral thigh flap for complex chest wall reconstruction: case report and review of the literature. Microsurgery 2016;36:70-6.

56. Taeger CD, Horch RE, Arkudas A, et al. Combined free flaps with arteriovenous loops for reconstruction of extensive thoracic defects after sternal osteomyelitis. Microsurgery 2016;36:121-7.

57. Wong CH, Senewiratne S, Garlick B, et al. Two-stage management of sternal wound infection using bilateral pectoralis major advancement flap. Eur J Cardiothorac Surg 2006;30: 148-52.

58. Davison SP, Clemens MW, Armstrong D, et al. Sternotomy wounds: rectus flap versus modified pectoral reconstruction. Plast Reconstr Surg 2007;120:929-34.

59. Yasuura K, Okamoto H, Morita S, et al. Results of omental flap transposition for deep sternal wound infection after cardiovascular surgery. Ann Surg 1998;227:455-9. 
60. Reade CC, Meadows WM Jr, Bower CE, et al. Laparoscopic omental harvest for flap coverage in complex mediastinitis. Am Surg 2003;69:1072-6.

61. Stump A, Bedri M, Goldberg NH, et al. Omental transposition flap for sternal wound reconstruction in diabetic patients. Ann Plast Surg 2010;65:206-10.

62. Saint-Cyr M, Wong C, Schaverien M, et al. The perforasome theory: vascular anatomy and clinical implications. Plast Reconstr Surg 2009; 124:1529-44.

63. Wettstein R, Weisser M, Schaefer DJ, et al. Superior epigastric artery perforator flap for sternal osteomyelitis defect reconstruction. J Plast Reconstr Aesthet Surg 2014;67:634-9.

64. Eburdery H, Grolleau JL, Berthier C, et al. Management of large sternal wound infections with the superior epigastric artery perforator flap. Ann Thorac Surg 2016;101:375-7.
65. Woo KJ, Pyon JK, Lim SY, et al. Deep superior epigastric artery perforator 'propeller' flap for abdominal wall reconstruction: a case report. J Plast Reconstr Aesthet Surg 2010;63: 1223-6.

66. Kannan RY. The internal mammary artery perforator flap and its subtypes in the reconstruction of median sternotomy wounds. J Thorac Cardiovasc Surg 2016;152:264-8.

67. Greig AV, Geh JL, Khanduja V, et al. Choice of flap for the management of deep sternal wound infection: an anatomical classification. J Plast Reconstr Aesthet Surg 2007;60: 372-8.

68. Weinand C, Phan TQV, Perbix W, et al. A reconstructive algorithm for deep sternal wound coverage: the Cologne-Merheim approach. Eur J Plast Surg 2013;36:95-104. 